\title{
Decay of excited surface electron states in liquid helium and related relaxation phenomena induced by short-wavelength ripplons
}

\author{
Yu.P. Monarkha, S.S. Sokolov, and A.V. Smorodin \\ B. Verkin Institute for Low Temperature Physics and Engineering of the National Academy of Sciences of Ukraine \\ 47 Lenin Ave., Kharkov 61103, Ukraine \\ E-mail: sokolov@ilt.kharkov.ua \\ Nelson Studart \\ Departamento de Física, Universidade Federal de São Carlos, 13565-905, São Carlos, São Paulo, Brazil
}

Received December 8, 2009, revised January 21, 2010

\begin{abstract}
Decay rates of excited surface electron states on liquid helium are theoretically studied for different electron confinement potentials and in the presence of quantizing magnetic field. Contributions of both one-ripplon and two-ripplon scattering processes are analyzed. Regarding the decay rate of the first excited surface level $(l=2)$, two-ripplon emission of short wave-length capillary waves is shown to dominate the conventional one-ripplon scattering in two distinct cases: the ambient temperature is low enough, or the surface state excitation energy $\Delta_{2}-\Delta_{1}$ does not match an excitation energy of the in-plane motion quantized under a strong magnetic field or in a quantum dot. In these cases, magnetic field and confinement cannot essentially reduce the decay rate which is of order of $10^{6} \mathrm{~s}^{-1}$ and does not depend on temperature. Importance of these findings for a microwave resonance experiment is discussed.
\end{abstract}

PACS: 73.20.-r Electron states at surfaces and interfaces;

73.20.-b Electron states and collective excitations in multilayers, quantum wells, mesoscopic, and nanoscale systems;

73.90.+f Other topics in electronic structure and electrical properties of surfaces, interfaces, thin films, and low-dimensional structures.

Keywords: liquid helium, surface electrons, microwave resonance, decay rate of excited states, quantum dot, quantum wire, two-ripplon scattering.

\section{Introduction}

Electrons trapped at the free surface of liquid helium can move freely along the interface forming a remarkable two-dimensional (2D) electron liquid (for reviews see $[1,2])$. At low electron densities the energy spectrum of the in-plane motion is continuous, $\varepsilon_{k}=\hbar^{2} k^{2} / 2 m$, where $\mathbf{k}$ is a $2 \mathrm{D}$ wave-vector, and $m$ is the free electron mass. In the direction normal to the interface, motion of surface electrons (SEs) is restricted to hydrogen-like bound states with energies $\Delta_{l}=-\Delta_{R} / l^{2}$, where $l$ is an integer $(l=1,2, \ldots)$ and $\Delta_{R}=m \Lambda^{2} / 2 \hbar^{2}$ is the effective Rydberg energy [here $\Lambda=e^{2}\left(\varepsilon_{\mathrm{He}}-1\right) / 4\left(\varepsilon_{\mathrm{He}}+1\right)$, and $\varepsilon_{\mathrm{He}}$ is the dielectric constant of liquid helium]. For liquid ${ }^{4} \mathrm{He}$ $\left(\varepsilon_{\mathrm{He}} \simeq 1.0572\right), \Delta_{R}$ is about $7.6 \mathrm{~K}$. The holding electric field $E_{\perp}$ applied usually in experiments on SEs shifts rela- tive positions of surface states similarly to the conventional Stark effect.

In recent years an essential attention is paid to the problem of the decay rate of excited surface states $(l>1)$ at low temperatures where electron scattering is determined by interaction with surface excitations of liquid helium (ripplons). The interest to this problem firstly arose because of the suggestion that SEs occupying two lowest Rydberg states with $l=1$ and $l=2$ can be used as electronic qubits controlled by a microwave (MW) field [3]. In this case the decay of the first excited level restricts the execution time of quantum computations. The decay rate affects also the linewidth of inter-subband transitions of SEs induced by MW radiation [4] and surface level occupancies $N_{l}$ at the MW resonance [5]. 
In the absence of magnetic field and at $T \gtrsim 0.1 \mathrm{~K}$, oneripplon scattering processes are quite sufficient for description of electron momentum relaxation, the decay rate of excited surface levels and the linewidth of the MW resonance. Electron scattering involving only one quantum of capillary waves is nearly elastic. The energy of ripplons involved $\hbar \omega_{q} \ll T$ and the average ripplon occupation number $N_{q} \simeq T / \hbar \omega_{q} \gg 1$ (here $\omega_{q}$ is ripplon dispersion) because the ripplon wave-vector $\mathbf{q}$ is restricted by electron wave-vector of the in-plane motion: $q \leq 2 k$. For liquid ${ }^{4} \mathrm{He}$ at $T \simeq 0.5 \mathrm{~K}$, the $\mathrm{SE}$ momentum relaxation rate is about $10^{7}-10^{8} \mathrm{~s}^{-1}$ and it is weakly decreasing with cooling. The above stated is true even for the decay rate of the excited surface levels $(l>1)$ because the energy difference between two levels is not given out but is transferred to the kinetic energy of electron motion along the interface. The later is the reason for decay heating of SEs in a MW resonance experiment $[5,6]$.

It should be noted that, for conventional one-ripplon scattering, low temperature behaviors of the momentum relaxation rate and the decay rate of the first excited surface level are quite different. For example, the holding field term of the interaction Hamiltonian $e E_{\perp} \xi(\mathbf{r})$ [here $e$ is electron charge and $\xi(\mathbf{r})$ is the surface displacement] leads to electron momentum relaxation proportional to $N_{q} / \varepsilon_{k} \propto T / \varepsilon_{k}$ [2] and, therefore, its contribution into the average momentum relaxation rate is independent of temperature. On the contrary, this term obviously does not contribute into the decay rate of excited surface states, while the contribution of other linear terms of the interaction Hamiltonian is proportional to $T /\left|\Delta_{l}-\Delta_{1}\right|$ which decreases steadily with cooling. The same is valid for the ripplon-limited width of MW resonance absorption. According to Ando [4], the diagonal matrix elements of the electron-ripplon coupling $U_{q}$ enter the ripplon-limited linewidth as the combination $\left(U_{q}\right)_{1,1}-\left(U_{q}\right)_{2,2}$ which is zero for the considering term $\left(U_{q} \rightarrow e E_{\perp}\right)$. Moreover, because of the strong cancellation of electron-ripplon interaction in such a combination of matrix elements, the main contribution into the ripplon-limited width of the MW resonance comes from the term which represents the decay rate of the excited surface state. Therefore, a theoretical analysis of contribution from interaction terms of higher orders (two-ripplon scattering) can be essential for understanding the low temperature behavior of the decay rate of excited surface states, the rate of information erasure in SE qubits, and the linewidth of MW resonance absorption.

In order to reduce the decay rate of the first exited Rydberg state of SEs it was proposed to separate electron energy levels applying a strong magnetic field in the direction normal to the surface or to use SEs confined in a small geometry (quantum dot system) [7]. In this case oneripplon scattering is strongly inelastic and the wavelength of ripplons involved is much shorter than the electron magnetic length $\left(L_{B}\right)$ or the electron localization radius
$\left(L_{c}\right)$ which makes the probability of scattering exponentially small. This was the reason for an optimistic estimate of the decay rate $v_{2 \rightarrow 1} \lesssim 10^{4} \mathrm{~s}^{-1}$ given previously [7] for $T \sim 0.1 \mathrm{~K}$. It is clear that such strong suppression of oneripplon scattering requires an accurate analysis of contributions from two-ripplon scattering events because for pairs of ripplons emitted by an electron in nearly opposite directions it is possible to conserve energy and momentum keeping the total momentum exchange small: $\left|\mathbf{q}^{\prime}+\mathbf{q}\right| \lesssim$ $\lesssim 1 / L_{c}, \quad 1 / L_{B} \ll q, q^{\prime}$. The importance of two-ripplon emission of short wavelength ripplons for SE energy relaxation and lifetime of excited Landau levels was proven already in 1978 [8]. Investigation of the lifetime of the first excited surface state for free SEs indicates that its low temperature asymptote is limited by spontaneous emission of pairs of short-wavelength surface excitations. The limiting value of the lifetime is about $10^{-6} \mathrm{~s}$, and it cannot be substantially increased by application of any strong magnetic field [9].

For confined SEs, two-ripplon scattering was considered previously only for nearly elastic processes, when an electron level of the in-plane quantization of the ground surface state is close to the corresponding level of the first excited surface state [7]. In this case only long-wavelengh ripplons with $q, q^{\prime}<1 / L_{c}$ are involved in scattering events. For such excitations, two-ripplon scattering probabilities of SEs are extremely small. At the same time, direct scattering between an excited surface state and the ground surface state involving pairs of short-wavelength ripplons with $q, q^{\prime}>10^{7} \mathrm{~cm}^{-1}$ was disregarded. Since surface excitations of superfluid helium with such large wave numbers are conventionally assumed to be well defined quasiparticles [10-12], their contribution into the decay rate of the excited states of confined SEs is of great importance.

Recently, the existence of remarkable magnetoconductivity oscillations induced by radiation and governed by the ratio of the radiation frequency $\omega=\left(\Delta_{2}-\Delta_{1}\right) / \hbar \equiv$ $\equiv \Delta_{21} / \hbar$ to the cyclotron frequency $\omega_{c}$ was demonstrated for SEs on liquid ${ }^{3} \mathrm{He}$ [13]. Magnetoconductivity $\sigma_{x x}$ is shown to be a periodic function of the parameter $\Delta_{21} / \hbar \omega_{c}$ having maxima when $\Delta_{21} / \hbar \omega_{c} \rightarrow$ integer (level matching conditions). In order to describe this effect one needs to know inter-level scattering rates at arbitrary relation between $\Delta_{21}$ and an excitation energy of the in-plane motion $\left(n^{\prime}-n\right) \hbar \omega_{c}$. As we shall see, a small mismatch between surface level excitation and Landau level excitation $\Delta_{21} \neq\left(n^{\prime}-n\right) \hbar \omega_{c}$ can suppress drastically conventional decay rates $v_{2 \rightarrow 1}$ even for the vapor atom scattering regime which requires inclusion into consideration inelastic two-ripplon scattering processes not affected significantly by the excitation energy mismatch.

In this work, we report results of theoretical investigation of the decay rate and inter-level scattering rates of excited surface states on liquid helium induced by two- 
ripplon scattering events involving short-wavelength ripplons $\left(q \gtrsim 10^{7} \mathrm{~cm}^{-1}\right)$ and compare them with results of conventional treatments. Special attention is given to the system of SEs confined in a small geometry. We discuss also the effects of two-ripplon scattering on observable properties of SEs such as the lifetime of a qubit state, linewidth of the MW resonance and even electron conductivity under MW excitation.

The article is organized as follows. In Sec. 2 we describe the approaches used to obtain the energy spectrum and wave functions of SE. In Sec. 3 we give the interaction Hamiltonians and expressions for the matrix elements of electron scattering. Some details are placed in Appendix. In Secs. 4-6 we calculate the decay rates of the excited SE surface state for 2D electron system, quantum dot, and quantum wire. The results of the work are summarized in Conclusion.

\section{Energy spectrum and wave functions}

To obtain SE wave functions in the presence of the perpendicular electric field $E_{\perp}$ a variational procedure is usually used. Trial wave functions are chosen to be of the same analytical form as those found explicitly for the case $E_{\perp}=0$ and $V_{0}=\infty$ [here $V_{0}$ is the repulsion barrier at the interface]. For two lowest surface levels, we assume

$$
\begin{gathered}
f_{1}(z)=A_{1} z \exp \left(-\gamma_{1} z\right) \\
f_{2}(z)=A_{2} z\left(1-\frac{\gamma_{12}}{3} z\right) \exp \left(-\gamma_{2} z\right)
\end{gathered}
$$

where $\gamma_{12}=\gamma_{1}+\gamma_{2}, \quad \gamma_{1}$ and $\gamma_{2}$ are variational parameters. The normalization constants are given by $A_{1}=2 \gamma_{1}^{3 / 2}$ and

$$
A_{2}=\frac{2 \sqrt{3} \gamma_{2}^{5 / 2}}{\left(\gamma_{1}^{2}-\gamma_{1} \gamma_{2}+\gamma_{2}^{2}\right)^{1 / 2}}
$$

It is convenient to use dimensionless variational parameters $x_{1}=\gamma_{1} / \gamma_{0}$ and $x_{2}=\gamma_{2} / \gamma_{0}$, where $\gamma_{0}=m \Lambda / \hbar^{2}$.

The surface level energies can be represented as

$$
\begin{gathered}
\Delta_{1}=\frac{\hbar^{2} \gamma_{0}^{2}}{2 m}\left[x_{1}\left(x_{1}-2\right)+\frac{2}{x_{1}}\left(\frac{\gamma_{\perp}}{\gamma_{0}}\right)^{3}\right], \\
\Delta_{2}=\frac{\hbar^{2} \gamma_{0}^{2}}{6 m\left(x_{1}^{2}-x_{1} x_{2}+x_{2}^{2}\right)} \times \\
\times\left[x_{2}^{2}\left(x_{1}^{2}-x_{1} x_{2}+7 x_{2}^{2}\right)-3 x_{2}\left(x_{1}^{2}-2 x_{1} x_{2}+3 x_{2}^{2}\right)+\right. \\
\left.+\frac{2}{x_{2}}\left(\frac{\gamma_{\perp}}{\gamma_{0}}\right)^{3}\left(5 x_{1}^{2}-2 x_{1} x_{2}+2 x_{2}^{2}\right)\right],
\end{gathered}
$$

with $\gamma_{\perp}^{3}=3 m e E_{\perp} /\left(2 \hbar^{2}\right)$. The parameters $x_{1}$ and $x_{2}$ are determined by the variation.

In the case of free SEs, the in-plane motion is described by the wave function $\varphi_{\mathbf{k}}(\mathbf{r})=S^{-1 / 2} \exp (i \mathbf{k} \cdot \mathbf{r})$ (here $S$ is the surface area occupied by electrons). The single-electron energy spectrum is given by $E_{l, k}=\Delta_{l}+\varepsilon_{k}$. For SEs under magnetic field $B$ oriented normally to the interface, $\varepsilon_{k}$ is replaced by the Landau spectrum $\varepsilon_{n}=\hbar \omega_{c}(n+1 / 2)$, where $\omega_{c}=e B / m c$ is the cyclotron frequency and $n=0,1,2, \ldots$. For SEs confined in a small geometry, we shall use an appropriate approximation for the wave functions and energy spectrum of the in-plane motion modelled by an harmonic potential.

\section{Interactions}

One-ripplon scattering is described by the interaction Hamiltonian linear in surface displacements:

$$
H_{e-r}^{(1)}=\frac{1}{\sqrt{S}} \sum_{\mathbf{q}} U_{q}(z) \xi_{\mathbf{q}} \exp (i \mathbf{q} \cdot \mathbf{r}) .
$$

Here $\xi_{\mathbf{q}}$ is the Fourier transformation of the surface displacement operator $\xi(\mathbf{r})$, and $U_{q}(z)$ is the electron-ripplon coupling. In the usual perturbation treatment (Bloch approach), the later can be presented in the following form [2]

$$
U_{q}(z)=e E_{q}(z)+e E_{\perp}-\frac{\partial V_{e}^{(0)}}{\partial z}
$$

where

$$
e E_{q}(z)=\Lambda q^{2}\left[\frac{1}{(q z)^{2}}-\frac{K_{1}(q z)}{q z}\right]
$$

is the polarization term, $V_{e}^{(0)}(z)$ is the total potential of a $\mathrm{SE}$ at the flat interface $(\xi=0)$, and $K_{n}(x)$ is the modified Bessel function of the second kind. The diagonal matrix elements of the last term are obviously zero $\left(-\partial V_{e}^{(0)} / \partial z\right)_{l, l}=0\left[\right.$ here $\left.(\hat{V})_{l, l^{\prime}}=\left\langle l^{\prime}|\hat{V}| l\right\rangle\right]$. The off-diagonal matrix elements can be rearranged as

$$
\left(-\frac{\partial V_{e}^{(0)}}{\partial z}\right)_{l, l^{\prime}}=\frac{\hbar^{2}}{2 m} f_{l}^{\prime}(0) f_{l^{\prime}}^{\prime}(0)-\left(\frac{\partial \mathrm{v}}{\partial z}\right)_{l, l^{\prime}},
$$

where $\mathrm{v}(z)=-\Lambda_{0} / z+e E_{\perp} z$, and $f_{l}^{\prime}(z)$ is the derivative of the SE wave function. It should be noted that $\mathrm{v}(z)$ is only an attractive part of $V_{e}^{(0)}(z)=V_{0} \theta(-z)+\mathrm{v}(z)$ with $\theta(x)$ being the unit step function. At $z<0$, there is the repulsion barrier $V_{0} \simeq 1 \mathrm{eV}$. In the limiting case $V_{0} \rightarrow \infty$ the values $f_{l}^{\prime}(0)$ can be expresses in terms of matrix elements of $\partial \mathrm{v} / \partial z[4]$ as

$$
f_{l}^{\prime}(0)=\frac{\sqrt{2 m}}{\hbar}\left(\frac{\partial \mathrm{v}}{\partial z}\right)_{l, l}^{1 / 2}
$$


so that the condition $\left(-\partial V_{e}^{(0)} / \partial z\right)_{l, l}=0$ is satisfied. It should be noted that one-ripplon scattering matrix elements are independent on which treatment (Bloch approach or adiabatic approximation) is used.

Two-ripplon scattering originates mostly from nonlinear (in $\xi_{\mathbf{q}}$ ) terms of the interaction Hamiltonian

$$
H_{e-r}^{(2)}=\frac{1}{S} \sum_{\mathbf{q}, \mathbf{q}^{\prime}} W_{\mathbf{q}, \mathbf{q}^{\prime}}(z) \xi_{\mathbf{q}} \xi_{\mathbf{q}^{\prime}} \exp \left[i\left(\mathbf{q}+\mathbf{q}^{\prime}\right) \cdot \mathbf{r}\right],
$$

because the electron potential at the interface has an extremely sharp structure. Next order terms of the perturbation treatment for the linear Hamiltonian (5) give much smaller probabilities of electron scattering in the ripplon wavelength range considered here. By now the two-ripplon coupling function $W_{\mathbf{q}, \mathbf{q}^{\prime}}(z)$ is found accurately only for two opposite limiting cases of short and long-wavelength ripplons. The matrix elements of two-ripplon scattering and energy conservation restrict the total momentum of a ripplon pair involved, and usually we have $\left|\mathbf{q}+\mathbf{q}^{\prime}\right| \ll q$. In this case the two-ripplon coupling depends mostly on $q \simeq q^{\prime}$, and we replace $W_{\mathbf{q}, \mathbf{q}^{\prime}}$ by a new function $W_{q}$.

In the limiting case of short-wavelength ripplons, the main contribution into $W_{q}$ comes from the interaction term $V_{0}[\theta(\xi-z)-\theta(-z)]$ whose important feature is that the matrix elements $\left\langle l^{\prime}\left|W_{q}\right| l\right\rangle$ does not depend on the ripplon wave number[8]

$$
\begin{aligned}
\left\langle l^{\prime}\left|W_{q}^{(\mathrm{sh})}\right| l\right\rangle & =\left(\frac{1}{2} \frac{\partial^{2} V_{e}^{(0)}}{\partial z^{2}}\right)_{l^{\prime}, l} \simeq V_{0} \kappa_{0}^{-1} f_{l}^{\prime}(0) f_{l^{\prime}}^{\prime}(0)= \\
& =\kappa_{0} \sqrt{\left(\frac{\partial \mathrm{v}}{\partial z}\right)_{l, l}\left(\frac{\partial \mathrm{v}}{\partial z}\right)_{l^{\prime}, l^{\prime}}}
\end{aligned}
$$

where $\kappa_{0}^{-1}=\hbar / \sqrt{2 m V_{0}} \simeq 2 \cdot 10^{-8} \mathrm{~cm}$ is the penetration length of the SE wave function into the liquid phase. Since $\left\langle l^{\prime}\left|W_{q}\right| l\right\rangle \sim \sqrt{V_{0}}$, this perturbation treatment cannot be used in the limiting case $V_{0} \rightarrow \infty$. In order to find the applicability range of Eq. (11), one should compare it with the result of the approximation $V_{0}=\infty$ analyzed previously [14].

For $V_{0}=\infty$, the electron wave function is zero at the interface $z=\xi(\mathbf{r})$. The replacement of the variable $z=\tilde{z}+\xi(\mathbf{r})$, which is analogous to an adiabatic treatment with the trial wave function $f_{l}(z-\xi)$, introduces the second order correction to the electron kinetic energy $p_{z}^{2}(\nabla \xi)^{2} / 2 m$. Gathering it and the second order terms of the polarization attraction one can find

$$
\left\langle l^{\prime}\left|W_{q}^{(\text {lon })}\right| l\right\rangle=\left(\frac{\hat{p}_{z}^{2}}{2 m}\right)_{l, l^{\prime}} q^{2}-\Lambda_{0}\left[\frac{2}{z^{3}}-\frac{q^{2}}{z} K_{2}(q z)\right]_{l, l^{\prime}} .
$$

Numerical calculations show that the second term of Eq. (12) is important only when $q \lesssim 2 \gamma_{1}$. It leads to an additional reduction in $\left\langle l^{\prime}\left|W_{q}^{(\text {lon })}\right| l\right\rangle$ in the long-wavelength limit. For $q \gg 2 \gamma_{1}$, the first term dominates being proportional to $q^{2}$.

Two-ripplon coupling of Eq. (12) does not depend on $V_{0}$, but it increases fast with the absolute value of the wave vector of ripplons involved in scattering events. Thus, the matrix elements of Eqs. (11) and (12), as functions of ripplon wave number, intersect at a certain value $q$ which equals $q_{0}^{*}=2 \sqrt{\kappa_{0} \gamma_{1}} \simeq 1.6 \cdot 10^{7} \mathrm{~cm}^{-1}$ for $l=l^{\prime}=1$. This means that the approximation $V_{0}=\infty$, or the trial wave function of the adiabatic approach $\left[f_{l}(z-\xi)\right]$, fails for $q>q_{0}^{*}$, because the wave function adjusting to the interface causes kinetic energy perturbations which are much higher than initial electron-ripplon interaction energies. Similarly, the short-wavelength asymptote of Eq. (11) is not applicable for $q<q_{0}^{*}$ because the adiabatic adjustment of the electron wave function leads to weaker scattering. Since it is impossible to describe two-ripplon coupling in the intermediate range ( $q \sim q_{0}^{*}$ ) we shall use the following interpolation

$$
W_{q}=W_{q}^{(\text {lon })} \theta\left(q_{0}^{*}-q\right)+W_{q}^{(\mathrm{sh})} \theta\left(q-q_{0}^{*}\right),
$$

where both $W_{q}^{(\text {lon) }}$ and $W_{q}^{(\text {sh) }}$ are considered as operators defined by their matrix elements given in Eqs. (12) and (11), respectively. Recently, such an approximation was shown to describe well the energy relaxation rate of electrons occupying the ground surface level in a nonlinear transport experiment [15].

\section{Decay rate of excited surface levels}

We shall define the decay rate $v_{l}$ of an excited surface level as a sum of transition rates $v_{l \rightarrow l^{\prime}}$ to all lower electron levels $\left(l^{\prime}<l\right)$ due to scattering. This quantity depends crucially on the properties of electron motion along the helium surface. Here we consider the following cases of electron confinement: 1) 2D electron system on a flat interface, including the case when a quantizing magnetic field $B$ is applied normally; 2) an electron is confined in a quantum dot; 3 ) electrons restricted to move freely in one-dimension only (quantum wire).

\section{1. $2 D$ electron system}

1) $B=0$. If there is no magnetic field applied, electron states bound to the interface are characterized by the surface level number $l$ and by the wave vector of the in-plane motion $\mathbf{k}$. For the interaction Hamiltonian of Eq. (10) and the displacement operator $\xi_{\mathbf{q}}=Q_{q}\left(b_{\mathbf{q}}+b_{-\mathbf{q}}^{\dagger}\right)$ [here $b_{\mathbf{q}}$ is the Bose destruction operator, $Q_{q}^{2}=\hbar q /\left(2 \rho \omega_{q} S\right)$, and $\rho$ is the liquid mass density], the decay rate due to emissions of two ripplons with nearly opposite momenta is 


$$
\begin{gathered}
v_{l}^{(2 r)}\left(\varepsilon_{k}\right)=\frac{4 \pi}{\hbar} \sum_{l<l} \sum_{\mathbf{q}, \mathbf{s}}\left|\left\langle l^{\prime}\left|W_{\mathbf{q}, \mathbf{q}-\mathbf{s} \mid}\right| l\right\rangle\right|^{2} \times \\
\times Q_{q}^{2} Q_{|\mathbf{q}-\mathbf{s}|}^{2}\left(N_{q}+1\right)\left(N_{|\mathbf{q}-\mathbf{s}|}+1\right) \\
\times \delta\left(\Delta_{l^{\prime}}-\Delta_{l}+\varepsilon_{|\mathbf{k}+\mathbf{s}|}-\varepsilon_{k}+\hbar \omega_{q}+\hbar \omega_{|\mathbf{q}-\mathbf{s}|}\right)
\end{gathered}
$$

where $\mathbf{s}=\mathbf{q}+\mathbf{q}^{\prime}$ is the total momentum of a ripplon pair, $N_{q}=\left[\exp \left(\hbar \omega_{q} / T\right)-1\right]^{-1}$. The familiar expression for ripplon frequency, $\omega_{q} \simeq \sqrt{\alpha / \rho} q^{3 / 2}$ (here $\alpha$ is the surface tension), is well-grounded for long wavelength excitations $q \sim 10^{4}-10^{6} \mathrm{~cm}^{-1}$. However, for short wavelength ripplons with $q \sim 10^{8} \mathrm{~cm}^{-1}$, strong deviations from the capillary spectrum are broadly discussed $[10,11,12]$.

For short-wavelength ripplons with $q>10^{7} \mathrm{~cm}^{-1}$, using energy conservation one can find that $s \ll q$. Then, the decay rate averaged over distribution of $\varepsilon_{k}$ reduces to

$$
\begin{array}{r}
\bar{v}_{l}^{(2 r)}=\frac{2 m S}{\hbar^{3}} \sum_{l^{\prime}<l \mathbf{q}} \sum_{\mid}\left|\left\langle l^{\prime}\left|W_{q}\right| l\right\rangle\right|^{2}\left(N_{q}+1\right)^{2} \times \\
\times Q_{q}^{4} \exp \left[-\frac{\left(2 \hbar \omega_{q}-\Delta_{l l^{\prime}}\right)}{T_{e}} \theta\left(2 \hbar \omega_{q}-\Delta_{l l^{\prime}}\right)\right],
\end{array}
$$

where $\Delta_{l l^{\prime}}=\Delta_{l}-\Delta_{l^{\prime}}$ and $T_{e}$ is the electron temperature. The structure of the exponential factor of this equation illustrates that for $T_{e} \ll\left|\Delta_{l l^{\prime}}\right|$ the energy of ripplon pairs most frequently emitted by electrons $2 \hbar \omega_{q} \lesssim\left|\Delta_{l l^{\prime}}\right|$.

We define $q_{l l^{\prime}}^{(2 r)}$ as the solution of the equation $2 \hbar \omega_{q}=\left|\Delta_{l l^{\prime}}\right|$. Consider the decay rate of the first excited surface level $\bar{v}_{2}^{(2 r)} \equiv \bar{v}_{2 \rightarrow 1}^{(2 r)}$. Assuming $\omega_{q}=\sqrt{\alpha / \rho} q^{3 / 2}$, we have

$$
q_{21}^{(2 r)}=\left(\frac{\rho}{4 \alpha}\right)^{1 / 3}\left(\frac{\Delta_{21}}{\hbar}\right)^{2 / 3}
$$

Even for the smallest value of $\Delta_{21} \simeq 5.7 \mathrm{~K}$ which is achieved at $E_{\perp}=0$, the typical wave number $q_{21}^{(2 r)} \simeq 3.8 \cdot 10^{7} \mathrm{~cm}^{-1}>q_{0}^{*}$. Therefore, $W_{q}$ of both regions $q<q_{0}^{*}$ and $q>q_{0}^{*}$ [see Eq. (13)] contributes to $v_{l}^{(2 r)}$. The contribution into $\bar{v}_{2 \rightarrow 1}^{(2 r)}$ from ripplons with $q>q_{0}^{*}$ can be found as

$$
\begin{gathered}
\frac{m \kappa_{0}^{2}}{4 \pi \rho \alpha \hbar}\left(\frac{\partial \mathrm{v}}{\partial z}\right)_{2,2}\left(\frac{\partial \mathrm{v}}{\partial z}\right)_{1,1} \times \\
\times\left[q_{21}^{(2 r)}-q_{0}^{*}+q_{21}^{(2 r)} \frac{2}{3}\left(\frac{T_{e}}{\Delta_{21}}\right)^{2 / 3} \int_{0}^{\infty} \frac{\exp (-x) d x}{\left(x+\Delta_{21} / T_{e}\right)^{1 / 3}} d q\right] .
\end{gathered}
$$

At low electron temperature $T_{e} \ll \Delta_{21}$ the third term in the square brackets of Eq. (17) can be disregarded. Then, gathering contributions from both regions of $q$ we obtain

$$
\bar{v}_{2 \rightarrow 1}^{(2 r)}=\frac{m\left(q_{0}^{*}\right)^{5}}{20 \pi \rho \alpha \hbar}\left(\frac{\hat{p}_{z}^{2}}{2 m}\right)_{1,2}^{2}+
$$

$$
+\frac{m \kappa_{0}^{2}}{4 \pi \rho \alpha \hbar}\left(\frac{\partial \mathrm{v}}{\partial z}\right)_{2,2}\left(\frac{\partial \mathrm{v}}{\partial z}\right)_{1,1}\left(q_{21}^{(2 r)}-q_{0}^{*}\right)
$$

Here the matrix element

$$
\left(\frac{\hat{p}_{z}^{2}}{2 m}\right)_{1,2}^{2}=\frac{16 \hbar^{4}}{3 m^{2}} \frac{\gamma_{1}^{5} \gamma_{2}^{5}}{\gamma_{12}^{4}\left(\gamma_{1}^{2}-\gamma_{1} \gamma_{2}+\gamma_{2}^{2}\right)}
$$

is calculated using the wave functions of Eqs. (1) and (2).

The first term of Eq. (18) represents the contribution from $W_{q}^{(\text {lon) }}$ given in Eq. (12), of which the second term is omitted because $q_{0}^{*}$ is substantially larger than $2 \gamma_{1}$. If we would use the approximation $f(z)=f_{l}(z-\xi)$ in the whole range of $q$ including $q \sim q_{21}^{(2 r)}$, then Eq. (18) would have only the first term of which the parameter $q_{0}^{*}$ should be replaced by $q_{21}^{(2 r)}$. In this case the decay rate $\bar{v}_{2 \rightarrow 1}^{(2 r)}$ would be much higher than the result given by Eq. (18), as shown in Fig. 1 by the dotted line. For pure Bloch treatment $\left(q_{0}^{*} \rightarrow 0\right)$, the decay rate is much lower (dashed line). Taking into account the long-wavelength reduction in $W_{q}$ which appears in the adiabatic (or $V_{0} \rightarrow \infty$ ) approximation we obtain a bit lower decay rate $\bar{v}_{2 \rightarrow 1}^{(2 r)}$ (solid line), which behaves similar to that found in the Bloch approach. Thus, for continuous spectrum of the in-plane motion of SEs, the long-wavelength reduction in $W_{q}$ discussed above leads only to inconsequential numerical correction to the result obtained previously. As we shall see, the situation will be different for a discrete energy spectrum $\varepsilon_{n}$ of the in-plane motion which appears under strong magnetic field or in the quantum dot system.

The result given in Eq. (18) represents the contribution from processes of spontaneous emission of ripplon pairs. It does not depend on temperature and, therefore, it can be considered as a lowest decay rate which can be achieved in

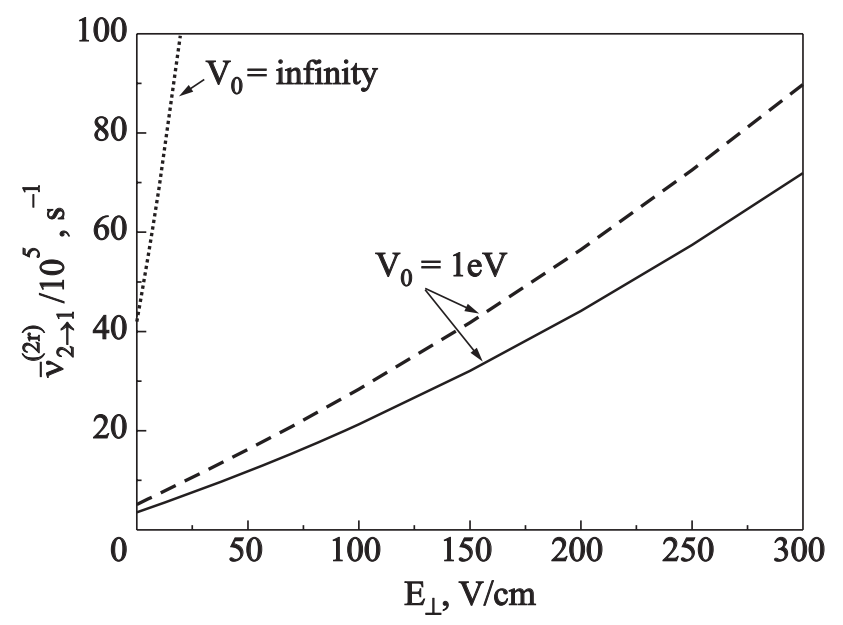

Fig. 1. Decay rates of the first excited surface level due to emission of pairs of short-wavelength ripplons for different theoretical models: approximation $V_{0}=\infty$ (dotted line), usual perturbation treatment $V_{0}=1 \mathrm{eV}$ (dashed line), combined model discussed in the text (solid line). 
experiments on SEs in liquid helium. At finite temperature one-ripplon scattering can contribute substantially. In order to estimate the importance of the result obtained above for typical experimental conditions, consider one-ripplon scattering described by the Hamiltonian given in Eq. (5). For quasi-elastic scattering ( $\hbar \omega_{q} \ll T$ ), we have

$$
\begin{aligned}
& v_{l}^{(1 r)}\left(\varepsilon_{k}\right) \simeq \frac{2 \pi}{\hbar} \sum_{l^{\prime}<l} \sum_{\mathbf{q}} Q_{q}^{2}\left|\left\langle l^{\prime}\left|U_{q}\right| l\right\rangle\right|^{2} \times \\
& \times\left(2 N_{q}+1\right) \delta\left(\Delta_{l}-\Delta_{l}+\varepsilon_{|\mathbf{k}+\mathbf{q}|}-\varepsilon_{k}\right),
\end{aligned}
$$

where $U_{q}(z)$ is defined in Eq. (6). Assuming $N_{q} \simeq T / \hbar \omega_{q} \gg 1$, after averaging over $\varepsilon_{k}$ we have

$$
\begin{gathered}
\bar{v}_{l}^{(1 r)}=\frac{T}{4 \sqrt{\pi} \alpha \hbar \sqrt{T_{e}}} \times \\
\times \sum_{l^{\prime}<l} \int_{0}^{\infty} \frac{d \varepsilon_{q}}{\varepsilon_{q}^{3 / 2}}\left|\left\langle l^{\prime}\left|U_{q}\right| l\right\rangle\right|^{2} \exp \left[-\frac{\left(\varepsilon_{q}-\Delta_{l l^{\prime}}\right)^{2}}{4 \varepsilon_{q} T_{e}}\right],
\end{gathered}
$$

where $\varepsilon_{q}=\hbar^{2} q^{2} / 2 m$. Because of the complicated $q$-dependence of $e E_{q}(z)$ given in Eq. (7), the integral of the right side of this expression can be evaluated only numerically using the explicit form of the matrix elements $\left\langle l^{\prime}\left|U_{q}\right| l\right\rangle$. For the first excited level $\bar{v}_{2}^{(1 r)}=\bar{v}_{2 \rightarrow 1}^{(1 r)}$, the straightforward transformation gives the following expression

$$
\begin{gathered}
\bar{v}_{2 \rightarrow 1}^{(1 r)}=\frac{4 T}{\sqrt{\pi}}\left\{\frac{\beta_{12}^{2} \gamma_{0}^{2} T_{e}}{\alpha \hbar} \int_{0}^{\infty} y^{2} \varphi_{12}^{2}\left(\frac{2 k_{T} y}{\gamma_{12}}\right) \times\right. \\
\times \exp \left[-\left(y-\frac{\Delta_{21}}{4 T_{e} y}\right)^{2}\right] d y+\frac{\beta_{12} \gamma_{0}\left(e E_{\perp}^{*}\right)}{2 \alpha \hbar} \int_{0}^{\infty} \varphi_{12}\left(\frac{2 k_{T} y}{\gamma_{12}}\right) \times \\
\left.\times \exp \left[-\left(y-\frac{\Delta_{21}}{4 T_{e} y}\right)^{2}\right] d y\right\}+\frac{T F_{12}^{2}}{2 \alpha \hbar \Delta_{21}}
\end{gathered}
$$

where $k_{T}=\sqrt{2 m T_{e}} / \hbar$ is electron thermal wave number and the rest of notations is given in Appendix.

The comparison between $\bar{v}_{2 \rightarrow 1}^{(1 r)}\left(E_{\perp}\right)$ and $\bar{v}_{2 \rightarrow 1}^{(2 r)}\left(E_{\perp}\right)$ as functions of the holding electric field is shown in Fig. 2 for different ambient temperatures. One can see that increasing $E_{\perp}$ extends the temperature region where $\bar{v}_{2 \rightarrow 1}^{(2 r)}$ dominates because $\bar{v}_{2 \rightarrow 1}^{(2 r)}$ increases faster with $E_{\perp}$ than $\bar{v}_{2 \rightarrow 1}^{(1 r)}$. This is in contrast with results obtained previously for intra-level collision frequency in which the one-ripplon contribution increases with $E_{\perp}$ much faster because of the interaction term $e E_{\perp} \xi$. Here for inter-level scattering the matrix elements of this interaction term are zero.

The quantity $v_{2 \rightarrow 1}^{(1 r)} / 2$ represents the decay rate contribution to the ripplon-limited width of the MW resonance absorption. The whole width $\gamma_{\text {op }}$ for optical transitions between levels $l=1$ and $l=2$ can be represented as a

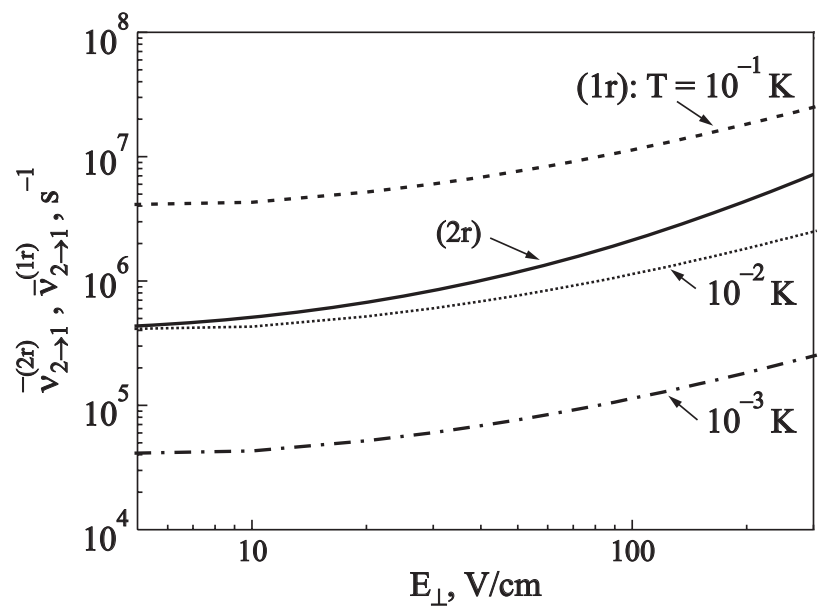

Fig. 2. The $\bar{v}_{2 \rightarrow 1}^{(1 r)}$ vs $E_{\perp}$ calculated for three ambient temperatures (dashed,dotted, and dash-dotted lines). Temperature independent $\bar{v}_{2 \rightarrow 1}^{(2 r)}$ is shown by the solid line.

sum of diagonal and off-diagonal terms [4] $\gamma_{\text {op }}=$ $=\gamma_{11-22}+\gamma_{21}$, where $\gamma_{21}=v_{2 \rightarrow 1}^{(1 r)}\left(\varepsilon_{k}\right) / 2$ and

$$
\gamma_{11-22}=\frac{\pi T}{\hbar \rho S} \sum_{\mathbf{q}} \frac{q}{\omega_{q}^{2}}\left[\left\langle 1\left|U_{q}\right| 1\right\rangle-\left\langle 2\left|U_{q}\right| 2\right\rangle\right]^{2} \delta\left(\varepsilon_{|\mathbf{k}+\mathbf{q}|}-\varepsilon_{k}\right) \text {. }
$$

Generally, $\gamma_{o p}$ is a function of electron energy of the in-plane motion $\varepsilon_{\mathbf{k}}$. Therefore we shall consider the averaged quantities $\bar{\gamma}_{21}=\bar{v}_{2 \rightarrow 1}^{(1 r)} / 2$ and $\bar{\gamma}_{11-22}$ which can be represented in the form similar to Eq. (21)

$$
\bar{\gamma}_{11-22}=\frac{T}{8 \sqrt{\pi} \alpha \hbar \sqrt{T_{e}}} \int_{0}^{\infty} \frac{d \varepsilon_{q}}{\varepsilon_{q}^{3 / 2}}\left[\left\langle 1\left|U_{q}\right| 1\right\rangle-\left\langle 2\left|U_{q}\right| 2\right\rangle\right]^{2} \mathrm{e}^{-\varepsilon_{q} / 4 T_{e}} .
$$

As already noted in the Introduction, the terms $e E_{\perp}$ and $-\partial V_{e}^{(0)} / \partial z$ entering into $U_{\mathbf{q}}$ give zero result for $\gamma_{11-22}$. Moreover, there is a strong cancellation of diagonal terms even for the residual coupling $e E_{q}(z)$. Therefore, the main contribution to one-ripplon broadening of the MW resonance comes from the off-diagonal term $\bar{\gamma}_{21}$ equal to the half of the decay rate $\bar{v}_{2 \rightarrow 1}^{(1 r)}$. This situation is illustrated in Fig. 3. Lines $\bar{\gamma}_{21}$ and $\bar{v}_{2 \rightarrow 1}^{(2 r)} / 2$ intersect approximately at $T \simeq 0.017 \mathrm{~K}$. This also allows us to speculate that at $T<0.017 \mathrm{~K}$, electron emission of pairs of short-wavelength ripplons will govern the ripplon-limited width of the MW resonance.

2) $B \neq 0$. In the presence of the magnetic field oriented in the normal direction to the surface, the electron spectrum of the in-plane motion is a set of equally spaced Landau levels $\left[\varepsilon_{n}=\hbar \omega_{c}(n+1 / 2)\right]$. In this case the decay rate of the first excited surface level $(l=2, n=0)$ can be written as a sum of decay transition rates to all lower states $l=1, n \leq n_{\max }$ (here $n_{\max }$ is the integer part of the ratio $\left.\Delta_{21} / \hbar \omega_{c}\right)$. For two-ripplon scattering, following Ref. 9, we can find 


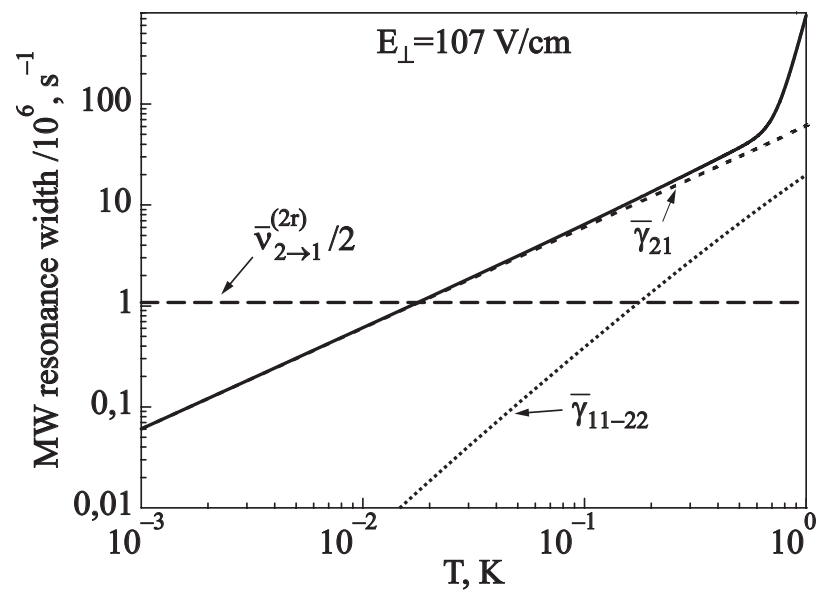

Fig. 3. The width of the MW resonance for electron scattering by ripplons and helium vapor atoms (solid) and the half of the decay rate of the first excited surface level induced by one-ripplon scattering (short-dashed line) and by two-ripplon emission processes (dashed line). The diagonal term $\bar{\gamma}_{11-22}$ is shown by the shortdotted line.

$$
v_{2 \rightarrow 1}^{(2 r)}(B)=\frac{1}{8 \pi L_{B}^{2} \rho^{2}} \sum_{n=0}^{n_{\max }}\left|\left\langle 1\left|W_{q_{n}}\right| 2\right\rangle\right|^{2} \frac{q_{n}^{3}}{\omega_{q_{n}}^{2}\left|\partial \omega_{q_{n}} / \partial q_{n}\right|}
$$

where $L_{B}=\sqrt{\hbar c / e B}$ is the magnetic length, $q_{n}$ is the root of the equation $2 \hbar \omega_{q}=\Delta_{21}-n \hbar \omega_{c}$. Using the new expression for two-ripplon coupling operator of Eq. (13), the decay rate can be found as

$$
\begin{aligned}
v_{2 \rightarrow 1}^{(2 r)}(B) & =\frac{1}{12 \pi L_{B}^{2} \alpha^{3 / 2} \rho^{1 / 2}} \sum_{n=0}^{n_{\max }}\left[\left(\frac{\hat{p}_{z}^{2}}{2 m}\right)_{1,2}^{2} q_{n}^{7 / 2} \theta\left(q_{0}^{*}-q_{n}\right)+\right. \\
& \left.+\kappa_{0}^{2}\left(\frac{\partial \mathrm{v}}{\partial z}\right)_{2,2}\left(\frac{\partial \mathrm{v}}{\partial z}\right)_{1,1} q_{n}^{-1 / 2} \theta\left(q_{n}-q_{0}^{*}\right)\right]
\end{aligned}
$$

This result indicates the importance of the long-wavelength $\left(q<q_{0}^{*}\right)$ reduction in the coupling operator $W_{q}$ given by the adiabatic approach with $f(z)=f_{l}(z-\xi)$. For usual perturbation treatment, $\quad v_{2 \rightarrow 1}^{(2 r)}(B) \propto 1 / q_{n}^{1 / 2} \rightarrow \infty$ when $n \hbar \omega_{c} \rightarrow \Delta_{21}\left(q_{n} \rightarrow 0\right)$, as illustrated in Fig. 4 by the dotted line. The new form of $W_{q}$ gives the decay rate which is finite even for $q_{n} \rightarrow 0$ (solid line). It should be noted that the sharp triangle-like bending of the solid line is the consequence of the interaction model [Eq. (13)]. For real interaction, they should be smoothed out. In the low field limit $(B \rightarrow 0)$, the solid line naturally approaches the result of Eq. (18) shown by the dashed line.

Consider now the one-ripplon scattering contribution to the electron decay rate under magnetic field $v_{2 \rightarrow 1}^{(1 r)}(B)$. If

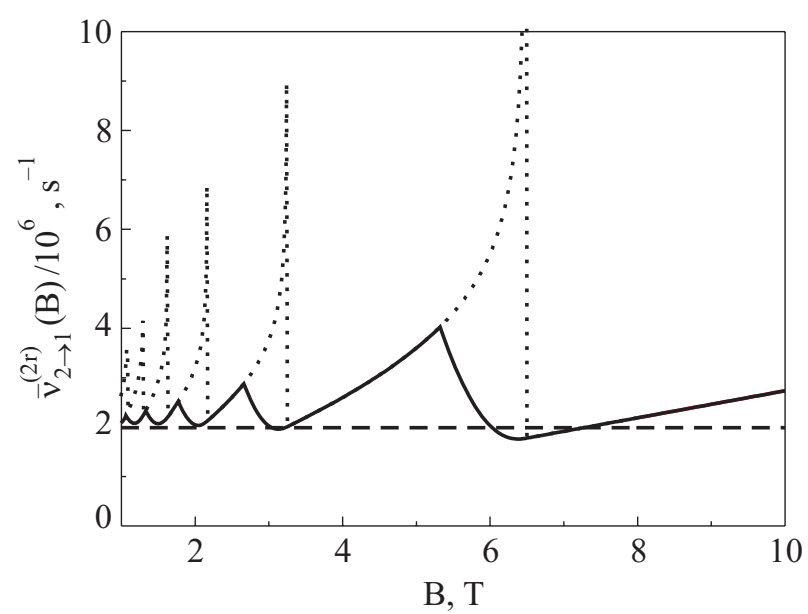

Fig. 4. The decay rate of the first excited surface level in the presence of strong magnetic field due to emission of pairs of short-wavelength ripplons for two theoretical models: usual perturbation treatment $V_{0}=1 \mathrm{eV}$ (dotted line), and the new model based on Eq. (13) (solid line).

$\Delta_{21}$ does not match the excitation spectrum of the in-plane motion $\left(n^{\prime}-n\right) \hbar \omega_{c}$ the energy conservation requires short wavelength excitations with $\omega_{q} \sim \omega_{c}$ to be involved in scattering. At the same time, momentum conservation which follows from matrix elements $\left(\mathrm{e}^{i \mathbf{q} \cdot \mathbf{r}}\right)_{X, n ; X^{\prime}, n^{\prime}}$ (here $X$ is the orbit center quantum number) restricts substantially the momentum exchange $q \lesssim L_{B}^{-1}$ because $\mid\left(\left.\exp \left(i q_{x} x\right)_{n ; n^{\prime}}\right|^{2} \propto \exp \left(-q^{2} L_{B}^{2} / 2\right)\right.$. Thus, one-ripplon scattering contribution into the decay rate $v_{2 \rightarrow 1}^{(1 r)}$ is exponentially small unless $\Delta_{21}$ is close enough to the matching condition $\left(n^{\prime}-n\right) \hbar \omega_{c}=\Delta_{21}$ when excitations with $q \lesssim L_{B}^{-1}$ can be involved in scattering. In this case, the singular nature of density of states (DOS) of noninteracting electrons is very important, and one should take into account the broadening of Landau levels $\Gamma_{l ; n}$ induced by scatterers. Here the subscript $l$ indicates that the strength of electron-ripplon interaction and the broadening depend on the surface level number. Thus, instead of the deltafunction of DOS of noninteracting electrons $\delta\left(\varepsilon-\varepsilon_{n}\right)$ we should use $-\operatorname{Im} G_{l, n}(\varepsilon) / \pi \hbar$, where $G_{l, n}(\varepsilon)$ the singleelectron Green's function. The cummulant expansion method [16] results in the Gaussian shape of Landau levels,

$$
-\operatorname{Im} G_{l, n}(\varepsilon)=\frac{\sqrt{2 \pi} \hbar}{\Gamma_{l ; n}} \exp \left[-\frac{2\left(\varepsilon-\varepsilon_{n}\right)^{2}}{\Gamma_{l, n}^{2}}\right] .
$$

The $\Gamma_{l, n}^{2}$ is usually expressed as the integral over $q$ whose integrand is proportional to $Q_{q}^{2}\left\langle l\left|U_{q}\right| l\right\rangle^{2}\left(2 N_{q}+1\right)$ [2].

In order to introduce the level broadening into the probability of electron scattering correctly, we shall use the way described in Ref. 17. In short, the probability should be rearranged into the form, containing the density of 
states of the initial and final states. Then the replacement $\delta\left(\varepsilon-\varepsilon_{n}\right) \rightarrow-\operatorname{Im} G_{l, n}(\varepsilon) / \pi \hbar$ is used. This simple selfconsistent way gives the correct result for magnetoconductivity in 2D electron systems which coincides with the result of more complicated treatment [18]. Thus, one can find

$$
\begin{aligned}
\bar{v}_{2 \rightarrow 1}^{(1 r)}= & \frac{2}{\pi \hbar^{3}} \sum_{n, n^{\prime} \mathbf{q}} \sum_{q} Q_{q}^{2}\left|\left(U_{q}\right)_{2,1}\right|^{2} J_{n, n^{\prime}}\left(q^{2} L_{B}^{2} / 2\right)\left(2 N_{q}+1\right) \times \\
& \times \int d \varepsilon w(\varepsilon) \operatorname{Im} G_{2, n}(\varepsilon) \operatorname{Im} G_{1, n^{\prime}}\left(\varepsilon+\Delta_{21}\right)
\end{aligned}
$$

where

$$
J_{n, n^{\prime}}(x)=\frac{\left[\min \left(n, n^{\prime}\right)\right] !}{\left[\max \left(n, n^{\prime}\right)\right] !} x^{\left|n-n^{\prime}\right|} \mathrm{e}^{-x}\left[L_{\min \left(n, n^{\prime}\right)}^{\left|n-n^{\prime}\right|}(x)\right]^{2},
$$

$L_{n}^{m}(x)$ are the associated Laguerre polynomials, and $w(\varepsilon)$ describes electron distribution over Landau levels.

For SEs on liquid helium, Landau levels are extremely narrow, $\Gamma_{l, n} \ll T, \hbar \omega_{c}$. In this case, we can replace $w(\varepsilon) \rightarrow w\left(\varepsilon_{n}\right)$ and find

$$
\begin{aligned}
& \int d \varepsilon^{\prime} \operatorname{Im} G_{2, n}\left(\varepsilon^{\prime}\right) \operatorname{Im} G_{1, n^{\prime}}\left(\varepsilon^{\prime}+\Delta_{21}\right)= \\
= & \frac{\pi^{3 / 2} \hbar^{2}}{\Gamma_{n, n^{\prime}}} \exp \left\{-\frac{\left[\Delta_{21}-\left(n^{\prime}-n\right) \hbar \omega_{c}\right]^{2}}{\Gamma_{n, n^{\prime}}^{2}}\right\},
\end{aligned}
$$

where $\Gamma_{n, n^{\prime}}^{2}=\left(\Gamma_{2 ; n}^{2}+\Gamma_{1 ; n^{\prime}}^{2}\right) / 2$. Then, the decay rate is

$$
\begin{gathered}
\bar{v}_{2 \rightarrow 1}^{(1 r)}=\frac{2 \pi^{1 / 2}}{\hbar Z_{\|}} \sum_{n, n^{\prime}} \mathrm{e}^{-n \hbar \omega_{c} / T_{e}} \times \\
\times \frac{1}{\Gamma_{n, n^{\prime}}} \exp \left\{-\frac{\left[\Delta_{21}-\left(n^{\prime}-n\right) \hbar \omega_{c}\right]^{2}}{\Gamma_{n, n^{\prime}}^{2}}\right\} \times \\
\times \sum_{\mathbf{q}} Q_{q}^{2}\left|\left(U_{q}\right)_{2,1}\right|^{2} J_{n, n^{\prime}}\left(q^{2} L_{B}^{2} / 2\right)\left(2 N_{q}+1\right),
\end{gathered}
$$

where $Z_{\|}=1 / 2\left[1+\operatorname{coth}\left(\hbar \omega_{c} / 2 T_{e}\right)\right]$. Thus, for strong magnetic fields $\Gamma_{n, n^{\prime}} \ll \hbar \omega_{c}<\Delta_{21}, \bar{v}_{21}^{(1 r)}(B)$ is exponentially small, except for very narrow regions where $\left(n^{\prime}-n\right) \hbar \omega_{c} \rightarrow \Delta_{21}$.

Similar and even more instructive equation can be found for the electron decay rate induced by scattering at helium vapor atoms $\bar{v}_{2 \rightarrow 1}^{(a)}$. Using the interaction pseudopotential of the contact type $\left[V_{\text {int }}^{(a)} \propto \delta\left(\mathbf{R}_{e}-\mathbf{R}_{a}\right)\right]$, we can easily integrate over $q$ and complete averaging over $n$ to obtain

$$
\begin{gathered}
\bar{v}_{2 \rightarrow 1}^{(a)}=v_{a}^{(0)} \frac{\hbar \omega_{c}}{\Gamma \pi^{1 / 2}} s_{2,1} \sum_{n=0}^{\infty} \exp \left[-\frac{\left[\Delta_{21}-n \hbar \omega_{c}\right]^{2}}{\Gamma^{2}}\right], \\
s_{l, l^{\prime}}=\frac{B_{1,1}}{B_{l, l^{\prime}}}, \quad B_{l, l^{\prime}}^{-1}=\int_{0}^{\infty} f_{l}(z)\left[f_{l^{\prime}}(z)\right]^{2} d z
\end{gathered}
$$

where $v_{a}^{(0)}$ is the electron collision frequency in the absence of magnetic field, $\Gamma=\left(2 / \pi \hbar^{2} \omega_{c} v_{a}^{(0)}\right)^{1 / 2}$ is the broadening induced by vapor atoms (it does not depend on $n$ ). Thus, close to the level matching conditions $\left(\left|\Delta_{21}-n \hbar \omega_{c}\right| \ll \Gamma\right)$ the decay rate is enhanced by the factor $\hbar \omega_{c} /\left(\Gamma \pi^{1 / 2}\right) \gg 1$ which represents the number of multiple scattering events within the lifetime. Beyond theses conditions $\left(\left|\Delta_{21}-n \hbar \omega_{c}\right|>\Gamma\right)$ the decay rate induced by vapor atoms $\bar{v}_{2 \rightarrow 1}^{(a)}$ is exponentially small, and therefore, even for high ambient temperatures $T \sim 1 \mathrm{~K}$ where vapor atoms scattering usually dominates, we should compare it with the contribution from two-ripplon scattering $\bar{v}_{2 \rightarrow 1}^{(2 r)}(B)$ found above.

The comparison between $\bar{v}_{2 \rightarrow 1}^{(a)}$ and $\bar{v}_{2 \rightarrow 1}^{(2 r)}$ is illustrated in Fig. 5 for $T=1 \mathrm{~K}$ and $E_{\perp} \simeq 100 \mathrm{~V} / \mathrm{cm}$. Once can see that $\bar{v}_{2 \rightarrow 1}^{(a)}$ is a periodic function of the parameter $\Delta_{21} / \hbar \omega_{c}$ with narrow peaks at $\Delta_{21} / \hbar \omega_{c} \rightarrow$ integer . Beyond these peaks it sharply decreases and becomes much less than the decay rate obtained for two-ripplon emission $\bar{v}_{2 \rightarrow 1}^{(2 r)}(B)$. Under the condition of this figure $\bar{v}_{2 \rightarrow 1}^{(1 r)}(B)$ can be disregarded because it has the same structure as $\bar{v}_{2 \rightarrow 1}^{(a)}(B)$ but with much smaller amplitude and broadening. Thus, under strong magnetic field even at high temperatures $T \sim 1 \mathrm{~K}$ there are regions where decay rate of the first excited surface level is determined by interaction with short wavelength ripplons.

If heating of the electron system is small, at the MW resonance the relative occupancy of the first excited surface level can be found using the two-level model [5]

$$
\frac{N_{2}}{N_{1}}=\frac{R_{m w}+\bar{v}_{2 \rightarrow 1} \exp \left(-\Delta_{21} / T_{e}\right)}{R_{m w}+\bar{v}_{2 \rightarrow 1}},
$$

where $R_{m w}$ is the absorption (emission) rate stimulated by $\mathrm{MW}$, and $\bar{v}_{2 \rightarrow 1}$ is the decay rate induced by all scattering processes discussed above. For vapor atom dominated re-

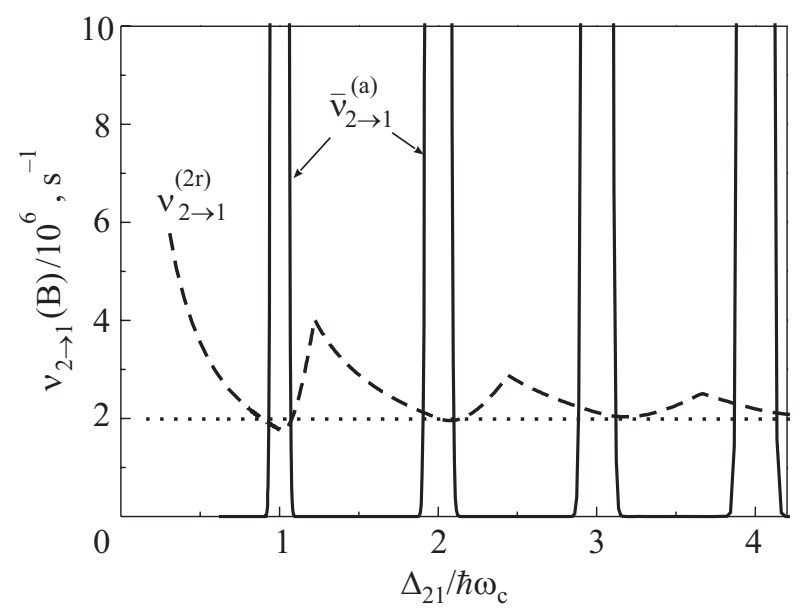

Fig. 5. Decay rates of the first excited surface level induced by vapor atoms $v_{2 \rightarrow 1}^{(a)}$ (solid) and by short wavelength ripplons $v_{2 \rightarrow 1}^{(2 r)}(B)$ (dashed) in presence of strong magnetic field. Dotted line represents $v_{2 \rightarrow 1}^{(2 r)}$ at $B=0$. 
gime $\bar{v}_{2 \rightarrow 1} \simeq \bar{v}_{2 \rightarrow 1}^{(a)}+\bar{v}_{2 \rightarrow 1}^{(2 r)}$. From Eqs. (30) and (32), we conclude that at sufficient MW excitation the occupancy of the second surface level is a periodic function of the parameter $\Delta_{21} / \hbar \omega_{c}$. It is large $\left(N_{2} / N_{1} \sim 1\right)$ in between of the level matching conditions where $\bar{v}_{2 \rightarrow 1}^{(a)} \rightarrow 0$ and it reduces fast when $\Delta_{21} / \hbar \omega_{c} \rightarrow$ integer because of the fast increase in $\bar{v}_{2 \rightarrow 1}^{(a)}$. This affects SE magnetoconductivity $\sigma_{x x}$ induced by vapor atom scattering because diagonal matrix elements $s_{l, l}$ for the ground and the first excited level differ substantially. For cold SEs $\left(T_{e}=T\right)$ variations in $\sigma_{x x}$ can be up to about $30 \%$. A strict description of magnetoconductivity oscillations requires heating of SEs to be taken into account and will be given elsewhere.

\section{Quantum dot}

According to the theoretical model $[3,7]$ and implementations [19], a SE qubit is formed by a single electron localized in $x-y$ plane due to the confining potential of the bottom electrode (quantum dot). Therefore, in this Section, we consider the decay rate of SE states assuming electron localization in the plane as well. Using a parabolic approximation for a confinement potential we classify the energy levels by the subscript $\{i\}=\left\{l, n_{x}, n_{y}\right\}$ as follows

$$
\begin{aligned}
& E_{l, n_{x}, n_{y}}=\Delta_{l}+\hbar \omega_{x}\left(n_{x}+\frac{1}{2}\right)+\hbar \omega_{y}\left(n_{y}+\frac{1}{2}\right) \\
& n_{x}, n_{y}=0,1,2 \ldots
\end{aligned}
$$

The oscillatory-like Hermite wave functions describe inplane $x-y$ motion of SEs, whereas the wave functions for the perpendicular motion are assumed to be the same as those of Eqs. (1) and (2).

Considering the problem of the decay rate in a quantum dot one should emphasize, as an essential point, that oneripplon processes give an exceptionally small contribution into $v_{l}^{(1 r)}$. Indeed, one can obtain the decay rate limited by one-ripplon processes for a state $\left\{l, n_{x}, n_{y}\right\}$ as

$$
\begin{gathered}
v_{\left(l, n_{x}, n_{y}\right)}^{(1 r)}=\frac{2 \pi}{\hbar} \sum_{l^{\prime}, n_{x}^{\prime}, n_{y}^{\prime}} \sum_{\mathbf{q}} Q_{q}^{2}\left|\left\langle l^{\prime}\left|U_{q}(z)\right| l\right\rangle\right|^{2} \times \\
\times J_{n_{x}^{\prime} n_{x}}\left(q_{x}^{2} L_{x}^{2} / 2\right) J_{n_{x}^{\prime} n_{x}}\left(q_{y}^{2} L_{y}^{2} / 2\right) \times \\
\times\left\{N_{q} \delta\left[\Delta_{l^{\prime}}-\Delta_{l}+\hbar \omega_{x}\left(n_{x}^{\prime}-n_{x}\right)+\hbar \omega_{y}\left(n_{y}^{\prime}-n_{y}\right)-\hbar \omega_{q}\right]+\right. \\
\left.+\left(N_{q}+1\right) \delta\left[\Delta_{l^{\prime}}-\Delta_{l}+\hbar \omega_{x}\left(n_{x}^{\prime}-n_{x}\right)+\hbar \omega_{y}\left(n_{y}^{\prime}-n_{y}\right)+\hbar \omega_{q}\right]\right\}
\end{gathered}
$$

where $L_{j}^{2}=\hbar /\left(m \omega_{j}\right)$ with $j$ equal $x$ or $y$, and $J_{n_{x}^{\prime} n_{x}}(x)$ is given by the Eq. (28).

As noted above the typical value of $\Delta_{2}-\Delta_{1} \sim 6 \mathrm{~K}$. If we do not consider the special case $\Delta_{l^{\prime} l}+\hbar \omega_{x}\left(n_{x}^{\prime}-n_{x}\right)+$ $+\hbar \omega_{y}\left(n_{y}^{\prime}-n_{y}\right) \rightarrow 0$ when the excitation energy of perpendicular motion matches the excitation energy of the inplane motion, for $\hbar \omega_{x} \sim \hbar \omega_{y}=0.1 \mathrm{~K}$ an analysis of the argument of the $\delta$-function gives typical values of $q>10^{7} \mathrm{~cm}^{-1}$, whereas $L_{j}>10^{-6} \mathrm{~cm}$. As a result, $J_{n_{x}^{\prime} n_{x}}\left(q_{j}^{2} L_{j}^{2} / 2\right)$ is extremely small and the one-ripplon contribution $v_{\left(l, n_{x}, n_{y}\right)}^{(1 r)}$ is negligible for any reasonable range of temperatures and holding fields. Under this condition only two-ripplon processes should be considered.

For two-ripplon emission, the decay rate of an excited surface level can be evaluated straightforwardly in the same way as Eq. (14):

$$
\begin{gathered}
v_{l}^{(2 r)}\left(\varepsilon_{n_{x}, n_{y}}\right) \simeq \frac{4 \pi}{\hbar} \sum_{l^{\prime}, n_{x}^{\prime}, n_{y}^{\prime}} \sum_{\mathbf{q}, \mathbf{s}} Q_{\mathbf{q}}^{4}\left|\left\langle l^{\prime}\left|W_{q}\right| l\right\rangle\right|^{2} \times \\
\times J_{n_{x}^{\prime} n_{x}}\left(s_{x}^{2} L_{x}^{2} / 2\right) J_{n_{x}^{\prime} n_{x}}\left(s_{y}^{2} L_{y}^{2} / 2\right) \times \\
\left.\times \delta \Delta_{l^{\prime}}-\Delta_{l}+\hbar \omega_{x}\left(n_{x}^{\prime}-n_{x}\right)+\hbar \omega_{y}\left(n_{y}^{\prime}-n_{y}\right)+2 \hbar \omega_{q}\right]
\end{gathered}
$$

where $s_{x} \ll q_{x}$ and $s_{y} \ll q_{y}$. For this reason, $J_{n_{x}^{\prime} n_{x}}\left(s_{x}^{2} L_{x}^{2} / 2\right)$ and $J_{n_{x}^{\prime} n_{x}}\left(s_{y}^{2} L_{y}^{2} / 2\right)$ can be of the order of unity which gives the decay rate comparable with the decay rate of free SEs considered in the previous Section.

With the approximation of $W_{q}$ given by Eq. (13), $v_{2 \rightarrow 1}^{(2 r)}$ of the quantum dot can be found as

$$
\begin{aligned}
& v_{2 \rightarrow 1}^{(2 r)}=\frac{1}{12 \pi L_{x} L_{y} \alpha^{3 / 2} \rho^{1 / 2}} \sum_{n_{x}^{\prime} n_{y}^{\prime}} \frac{I_{n_{x}^{\prime} n_{x}} I_{n_{y}^{\prime} n_{y}}}{\sqrt{q_{\mathbf{n}, \mathbf{n}^{\prime}}}} \times \\
& \quad \times\left[\left(\frac{p_{z}^{2}}{2 m}\right)_{1,2}^{2} q_{\mathbf{n}, \mathbf{n}^{\prime}}^{4} \theta\left(q_{0}^{*}-q_{\mathbf{n}, \mathbf{n}^{\prime}}\right)+\right. \\
& \left.+\kappa_{0}^{2}\left(\frac{\partial \mathbf{v}}{\partial z}\right)_{2,2}\left(\frac{\partial \mathbf{v}}{\partial z}\right)_{1,1} \theta\left(q_{\mathbf{n}, \mathbf{n}^{\prime}}-q_{0}^{*}\right)\right],
\end{aligned}
$$

where

$$
q_{\mathbf{n}, \mathbf{n}^{\prime}}=\left(\frac{\rho}{4 \alpha \hbar^{2}}\right)^{1 / 3}\left[\Delta_{21}+\hbar \omega_{x}\left(n_{x}-n_{x}^{\prime}\right)+\hbar \omega_{y}\left(n_{y}-n_{y}^{\prime}\right)\right]^{2 / 3} .
$$

The summation is carried out over all $n_{x}^{\prime}$ and $n_{y}^{\prime}$ satisfying the condition $\Delta_{21}+\hbar \omega_{x}\left(n_{x}-n_{x}^{\prime}\right)+\hbar \omega_{y}\left(n_{y}-n_{y}^{\prime}\right)>$ $>0$, and

$$
I_{n_{j}^{\prime} n_{j}}=\int_{0}^{\infty} x^{-1 / 2} J_{n_{j}^{\prime}, n_{j}}(x) d x
$$

For $q_{\mathbf{n}, \mathbf{n}^{\prime}}>q_{0}^{*}$, Eq. (36) transforms into the result briefly reported in Ref. 20. Still, one should take into account the first term when $\Delta_{21}+\hbar \omega_{x}\left(n_{x}-n_{x}^{\prime}\right)++\hbar \omega_{y}\left(n_{y}-n_{y}^{\prime}\right)$ strongly decreases to avoid the divergence $1 / \sqrt{q_{\mathbf{n}, \mathbf{n}^{\prime}}}$.

In Fig. 6, $v_{2 \rightarrow 1}^{(2 r)}$ is plotted as a function of the holding electric field. A weak oscillatory dependence of $v_{2 \rightarrow 1}^{(2 r)}\left(E_{\perp}\right)$ in standard perturbation treatment $\left(q_{\mathbf{n}, \mathbf{n}^{\prime}}>q_{0}^{*}\right)$ is due to approaching the condition $q_{\mathbf{n}, \mathbf{n}^{\prime}} \rightarrow 0$ for a given value of $\Delta_{21}$ when varying $E_{\perp}$ [20]. The modification of 


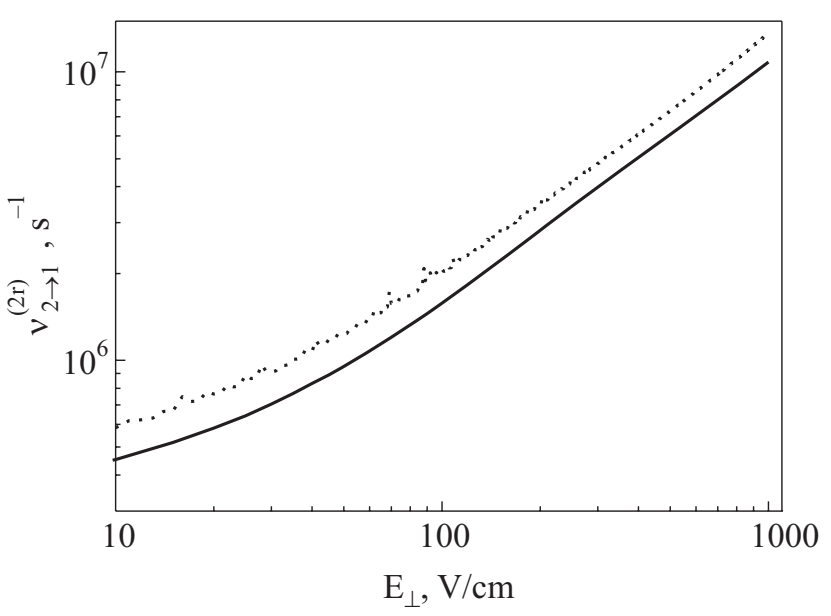

Fig. 6. The $v_{2 \rightarrow 1}^{(2 r)}$ as a function of the holding electric field for $\hbar \omega_{x}=0.6 \mathrm{~K}, \hbar \omega_{y}=0.7 \mathrm{~K}$ and $n_{x}=n_{y}=1:$ usual perturbation treatment (dotted line) and the model based on Eq. (13) (solid line).

the long-wavelength $\left(q_{\mathbf{n}, \mathbf{n}^{\prime}}<q_{0}^{*}\right)$ part of $W_{q}$ discussed above eliminates singularities $1 / \sqrt{q_{\mathbf{n}, \mathbf{n}^{\prime}}}$ of the two-ripplon coupling function (straight line). The Fig. 6 indicates also that the decay rate of the first excited surface level in a quantum dot $v_{2 \rightarrow 1}^{(2 r)}$ is of the same order as that shown in Fig. 1 for free SEs.

\section{Quantum wire}

Consider a quasi-one-dimensional electron system of which electrons are free to move in one in-plane direction only, whereas their motion across this direction is restricted (quantum wire). For such a SE system, the energy levels $\{i\}=\left\{l, k_{x}, n\right\}$ are given by

$$
E_{l, n, k_{x}}=\Delta_{l}+\varepsilon_{k_{x}}+\hbar \omega_{0}\left(n+\frac{1}{2}\right),
$$

where a parabolic confinement is assumed, $n=0,1,2 \ldots$, and $\varepsilon_{k_{x}}=\hbar^{2} k_{x}^{2} / 2 m$. Practically, the confinement potential is created by a distortion of the liquid helium surface over a specially created substrate due to capillary forces [21]. In this case $\omega_{0}$ is a function of the curvature radius $R$ and the holding electric field:

$$
\omega_{0}=\left(\frac{e E_{\perp}}{m R}\right)^{1 / 2} .
$$

Under the approximation, the wave functions of the oscillatory part of the energy spectrum of Eq. (38) are given by Hermite functions. If SE confinement is created by an external potential using a system of specially arranged electrodes, the frequency $\omega_{0}$ will be dependent on the magnitude of the potentials applied to these electrodes.
In a quantum wire, the decay rate for two-ripplon processes can be written as

$$
\begin{aligned}
& v_{l}^{(2 r)}\left(k_{x}, n\right) \simeq \frac{4 \pi}{\hbar} \sum_{l^{\prime} n^{\prime}} \sum_{\mathbf{q}, \mathbf{s}} Q_{q}^{4}\left|\left\langle l^{\prime}\left|W_{q}\right| l\right\rangle\right|^{2} J_{n^{\prime} n}\left(s_{y}^{2} L_{y}^{2} / 2\right) \times \\
& \times \delta\left[\Delta_{l^{\prime}}-\Delta_{l}+\hbar \omega_{0}\left(n^{\prime}-n\right)+\varepsilon_{k_{x}+s_{x}}-\varepsilon_{k_{x}}+2 \hbar \omega_{q}\right] .(40)
\end{aligned}
$$

Averaging this equation over Boltzmann distribution of $\varepsilon_{k_{x}}$ results in an expression for a decay rate $v_{l}^{(2 r)}(n)$ depending on two discrete quantum numbers $l$ and $n$ (here we separate the in-plane number $n$ by placing it in round brackets). For the first excited surface level, $v_{2}^{(2 r)}(n)=v_{2 \rightarrow 1}^{(2 r)}(n)$ is found as

$$
\begin{aligned}
v_{2 \rightarrow 1}^{(2 r)}(n) & =\frac{\Gamma(2 / 3)}{\sqrt{2} \pi^{3 / 2} \Gamma(1 / 6)} \frac{m^{1 / 2}}{\hbar^{1 / 2} \alpha^{5 / 4} \rho^{3 / 4} l_{0}} \sum_{n^{\prime}}\left(q_{21}^{n n^{\prime}}\right)^{1 / 4} I_{n n^{\prime}} \times \\
\times & \left\{\frac{\Gamma(10 / 3) \Gamma(1 / 6)}{6 \Gamma(23 / 6) \Gamma(2 / 3)}\left(\frac{p_{z}^{2}}{2 m}\right)_{1,2}^{2}\left(q_{21}^{n n^{\prime}}\right)^{4} \theta\left(q_{0}^{*}-q_{21}^{n n^{\prime}}\right)+\right. \\
+ & {\left[\kappa_{0}^{2}\left(\frac{\partial \mathrm{v}}{\partial z}\right)_{2,2}\left(\frac{\partial \mathrm{v}}{\partial z}\right)_{1,1}\left[1-B_{0}\left(\frac{q_{0}}{q_{21}^{n n^{\prime}}}\right)\right]+\right.} \\
+ & \left.\left.B_{4}\left(\frac{q_{0}}{q_{21}^{n n^{\prime}}}\right)\left(\frac{p_{z}^{2}}{2 m}\right)_{1,2}^{2}\left(q_{21}^{n n^{\prime}}\right)^{4}\right] \theta\left(q_{21}^{n n^{\prime}}-q_{0}^{*}\right)\right\},
\end{aligned}
$$

where $l_{0}=\left[\hbar /\left(m \omega_{0}\right)\right]^{1 / 2}, I_{n n^{\prime}}$ is given by Eq. (37),

$$
\begin{aligned}
& q_{21}^{n n^{\prime}}=\left(\frac{\rho}{4 \alpha \hbar^{2}}\right)^{1 / 3}\left[\Delta_{21}+\hbar \omega_{0}\left(n-n^{\prime}\right)\right]^{2 / 3} \\
& B_{\lambda}(y)=\frac{\Gamma(1 / 6)}{4 \sqrt{\pi} \Gamma(2 / 3)} \int_{0}^{y} \frac{t^{\lambda} d t}{\sqrt{1-t^{3 / 2}}} .
\end{aligned}
$$

Equation (41) obtained for two-ripplon coupling operator of Eq. (13) is valid for $T_{e} \ll \Delta_{21}+\hbar \omega_{0}\left(n-n^{\prime}\right)$. The summation is carried out over all $n^{\prime}$ satisfying the condition of positiveness of $\Delta_{21}+\hbar \omega_{0}\left(n-n^{\prime}\right)$ for given $n$. The result of usual perturbation treatment obtained previously follows from Eq. (41) as the limiting case $q_{0}^{*} \rightarrow 0$.

In analogy with a free $2 \mathrm{D}$ electron system, one can expect a substantial contribution from one-ripplon scattering into the decay rate of a quantum wire at high enough temperatures. Following the standard procedure one can find the result similar to that of Eq. (20):

$$
\begin{aligned}
& v_{l}^{(1 r)}\left(k_{x}, n\right) \simeq \frac{2 \pi}{\hbar} \sum_{l^{\prime}<l} \sum_{\mathbf{q}} Q_{\mathbf{q}}^{2}\left|\left\langle l^{\prime}\left|U_{\mathbf{q}}\right| l\right\rangle\right|^{2} J_{n^{\prime} n}\left(q_{y}^{2} L_{y}^{2} / 2\right) \times \\
& \times\left(2 N_{\mathbf{q}}+1\right) \delta\left[\Delta_{l^{\prime}}-\Delta_{l}+\hbar \omega_{0}\left(n^{\prime}-n\right)+\varepsilon_{k_{x}+q_{x}}-\varepsilon_{k_{x}}\right] .
\end{aligned}
$$


After averaging over $\varepsilon_{k x}$ we arrive at the following decay rate of the quantum wire induced by one-ripplon scattering processes:

$$
\begin{gathered}
v_{2 \rightarrow 1}^{(1 r)}(n)=\frac{T}{\pi^{3 / 2} \alpha \hbar Z_{\|} \sqrt{\hbar \omega_{0} T_{e}}} \times \\
\times \sum_{n^{\prime}} \int_{0}^{\infty} \frac{d x}{x} \exp \left[-\frac{\hbar \omega_{0}}{4 T_{e}}\left(x-\frac{\Delta_{21} / \hbar \omega_{0}+n-n^{\prime}}{x}\right)^{2}\right] \times \\
\times \int_{0}^{\infty} \frac{d y}{\left(x^{2}+y^{2}\right)} J_{n n^{\prime}}\left(y^{2}\right) \times \\
\times\left[\beta_{12} \gamma_{0}\left(\hbar \omega_{0}\right)\left(x^{2}+y^{2}\right) \varphi_{12}\left(\frac{\gamma_{0}}{\gamma_{12}} \sqrt{\frac{\hbar \omega_{0}\left(x^{2}+y^{2}\right)}{\Delta_{0}}}\right)+F_{12}\right]^{2},
\end{gathered}
$$

where now $Z_{\|}=1 / 2\left[1+\operatorname{coth}\left(\hbar \omega_{0} / 2 T_{e}\right]\right.$, and other notations are the same as those in Eq. (21) and in Appendix.

The comparison between the conventional contribution into the electron decay rate $v_{2 \rightarrow 1}^{(1 r)}$ and the corresponding contribution from short-wavelength ripplons $v_{2 \rightarrow 1}^{(2 r)}$ is shown in Fig. 7 for a fixed value of the confinement frequency $\omega_{0}$. In the case of a fixed curvature radius $R$ with $\omega_{0}$ given by Eq. (39), the results of numerical calculations give similar results with some lower curves which is inconsequential for our analysis. The curves of Fig. 7 are plotted for high enough holding fields $E_{\perp}$ where the square of the transverse oscillation mode frequency is positive [22] which provides the stability of the system. One can see that for such strong holding fields, $v_{2 \rightarrow 1}^{(2 r)}$ dominates the conventional contribution already at $T \sim 0.1 \mathrm{~K}$ due to a more strong field dependence already noticed above for the free 2D electron system on liquid helium. It should be noted that regarding the decay rate of excited

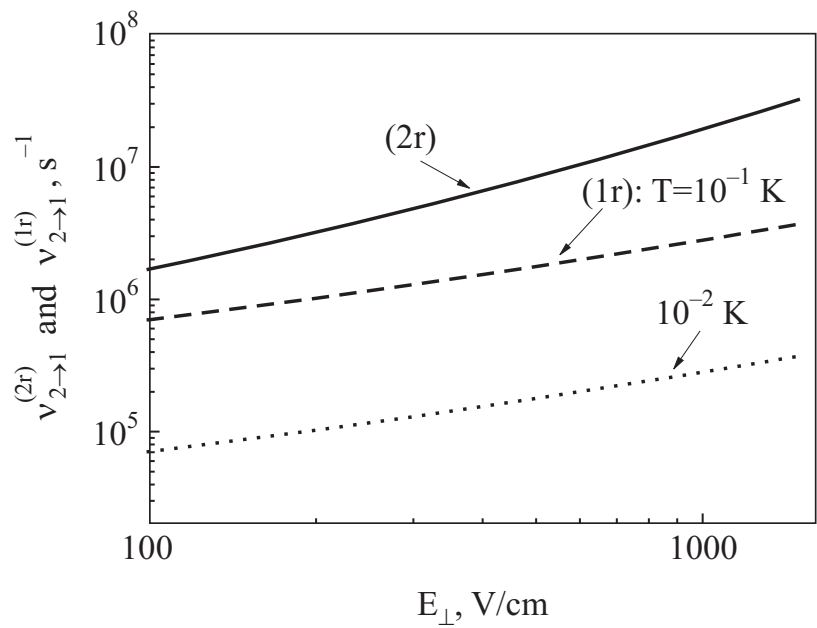

Fig. 7. The decay rate $v_{2 \rightarrow 1}^{(1 r)}$ of the quantum wire vs $E_{\perp}$ calculated for three ambient temperatures (dashed and dotted), $n=1$ and $\hbar \omega_{0}=0.5 \mathrm{~K}$. Temperature independent $v_{2 \rightarrow 1}^{(2 r)}$ is shown by the solid line. surface levels the quantum wire is similar rather to free SEs than to a quantum dot system or SEs under quantizing magnetic field, because of the continuous component of the electron spectrum $\varepsilon_{k_{x}}$.

\section{Conclusion}

In the present work we theoretically studied the decay rate of excited surface electron states in liquid helium under different conditions with regard to external fields and confinement potentials which are usually used in experiments with SEs especially in those where qubits are modelled. In addition to conventional one-ripplon processes involving only long wavelength excitations, two-ripplon emission of short wavelength capillary waves is considered taking into account the reduction in the coupling operator due to adiabatic adjustment of SE wave functions to the interface. This reduction is shown to be very important for SEs under a strong magnetic field oriented in the normal direction to the interface or in a quantum dot system, because it eliminates singular behavior of the decay rate when the in-plane excitation energy $\varepsilon_{n^{\prime}}-\varepsilon_{n}$ approaches the excitation energy of surface levels $\Delta_{21}$.

For all confinement potentials and external fields considered here, the decay rate of the first excited surface level induced by emission of short wavelength ripplons is found to be independent of temperature. Therefore, at low enough temperatures it dominates the conventional oneripplon contribution which is proportional to the average ripplon occupation number $N_{q} \propto T$. The characteristic temperature where both these contributions are of the same order depends on the holding electric field and varies from about $0.01 \mathrm{~K}$ to about $0.1 \mathrm{~K}$ for typical experimental conditions. The lowest decay rate $v_{2 \rightarrow 1}^{(2 r)} \simeq 4 \cdot 10^{5} \mathrm{~s}^{-1}$ is obtained for zero holding electric field. This number can be considered as the lowest rate of information erasure which can be achieved for a qubit formed by an electron occupying two lowest surface levels over superfluid helium.

The analysis given here shows also that in the presence of a strong magnetic field or in a quantum dot system, the temperature region where the electron decay rate induced by short wavelength ripplons dominates can be extended up to high temperatures $T \sim 1 \mathrm{~K}$. This happens when the surface state excitation energy $\Delta_{21}$ does not match the inplane excitation energy $\varepsilon_{n^{\prime}}-\varepsilon_{n}$. In particular, under magnetic field the vapor-atom induced decay rate is found to be a sharp oscillatory function of the parameter $\Delta_{21} / \hbar \omega_{c}$. It exceeds the corresponding contribution from short wavelength ripplons only in the vicinity of the level matching conditions: $\Delta_{21} / \hbar \omega_{c} \rightarrow$ integer. These matching conditions are restricted by the Landau level broadening which is much smaller than $\hbar \omega_{c}$. Therefore, even in the high temperature regime, where electron conductivity is governed by scattering at vapor atoms, the decay rate of ex- 
cited surface levels induced by interaction with short wavelength ripplons can determine level occupancies of SEs in a MW resonance experiment.

It should be noted that short wavelength excitations of a liquid helium surface with $q \sim 5 \cdot 10^{7} \mathrm{~cm}^{-1}$ are well defined quasi-particles only for liquid ${ }^{4} \mathrm{He}$. In the case of liquid ${ }^{3} \mathrm{He}$, damping effects are very strong already for capillary waves with $q \sim 10^{5} \mathrm{~cm}^{-1}$. Therefore, the results obtained here for SEs over superfluid ${ }^{4} \mathrm{He}$ cannot be applied directly for SEs formed on liquid ${ }^{3} \mathrm{He}$. Still, it is known experimentally that ripplon-limited mobility of SEs over liquid ${ }^{3} \mathrm{He}$ is not affected by strong damping effects. This allows us to speculate that generally two-ripplon decay rate of the first excited surface level over liquid ${ }^{3} \mathrm{He}$ should be the same as that found here. Anyway, an experimental observation of a significant difference in the decay rate and surface level occupation under MW resonance for SEs formed on such a Fermi-liquid substrate would be of great importance.

This work was partly supported by STCU through Project 3718. One of us (N.S.) is supported by a grant from CNPq.

\section{Appendix}

Here we give the expressions for the matrix elements of one-ripplon scattering processes.

The interaction Hamiltonian for the electron scattering by one-ripplon is given by

$$
\hat{H}^{(1 r)}(\mathbf{r}, z, t)=\sum_{\mathbf{q}} Q_{\mathbf{q}} U_{q}(z)\left(a_{\mathbf{q}} \mathrm{e}^{i\left(\mathbf{q} \cdot \mathbf{r}-\omega_{\mathbf{q}} t\right)}+a_{\mathbf{q}}^{\dagger} \mathrm{e}^{-i\left(\mathbf{q} \cdot \mathbf{r}-\omega_{\mathbf{q}} t\right)}\right) .
$$

The matrix elements can be written as [2]

$$
\begin{aligned}
\left\langle l^{\prime}\left|U_{q}(z)\right| l\right\rangle & =\Lambda_{0}\left\langle l^{\prime}\left|\frac{1}{z^{2}}-\frac{q}{z} K_{1}(q z)\right| l\right\rangle+e E_{\perp} \delta_{l l^{\prime}}+F_{l^{\prime} l} \\
F_{l^{\prime} l} & =\sqrt{\left(\frac{\partial \mathrm{v}}{\partial z}\right)_{l l}\left(\frac{\partial \mathrm{v}}{\partial z}\right)_{l^{\prime} l^{\prime}}}-\left(\frac{\partial \mathrm{v}}{\partial z}\right)_{l l^{\prime}} .
\end{aligned}
$$

The last term is essential for inter-subband $(l \neq l)$ scattering only.

If we consider $l, l^{\prime}=1,2$ and apply the trial wave functions of Eqs. (1) and (2) we obtain

$$
\begin{gathered}
\left\langle 1\left|U_{q}(z)\right| 1\right\rangle=\frac{\Lambda_{0} q^{2}}{2} \varphi_{11}\left(\frac{q}{2 \gamma_{1}}\right)+e E_{\perp} ; \\
\left\langle 2\left|U_{q}(z)\right| 2\right\rangle=\frac{\Lambda_{0} q^{2}}{2} \beta_{22} \varphi_{22}\left(\frac{q}{2 \gamma_{2}}\right)+e E_{\perp} ; \\
\left\langle 1\left|U_{q}(z)\right| 2\right\rangle=\frac{\Lambda_{0} q^{2}}{2} \beta_{12} \varphi_{12}\left(\frac{q}{\gamma_{12}}\right)+F_{12} ;
\end{gathered}
$$

with

$$
\begin{gathered}
\left(\frac{\partial \mathrm{v}}{\partial z}\right)_{11}=2 \Lambda_{0} \gamma_{1}^{2}+e E_{\perp} \\
\left(\frac{\partial \mathrm{v}}{\partial z}\right)_{22}=2 \beta_{22}\left(1-\frac{\gamma_{12}}{3 \gamma_{2}}+\frac{\gamma_{12}^{2}}{18 \gamma_{2}^{2}}\right) \Lambda_{0} \gamma_{2}^{2}+e E_{\perp} \\
\left(\frac{\partial \mathrm{v}}{\partial z}\right)_{21}=\beta_{12} \Lambda_{0} \gamma_{12}^{2}
\end{gathered}
$$

The parameters $\beta^{\prime}$ s depend on the $\gamma^{\prime}$ s according to the relations

$$
\begin{gathered}
\beta_{12}=\frac{8}{\sqrt{3}} \frac{\gamma_{1}^{3 / 2} \gamma_{2}^{5 / 2}}{\gamma_{12}^{3}\left(\gamma_{1}^{2}-\gamma_{1} \gamma_{2}+\gamma_{2}^{2}\right)^{1 / 2}} \\
\beta_{22}=\frac{3 \gamma_{2}^{2}}{\left(\gamma_{1}^{2}-\gamma_{1} \gamma_{2}+\gamma_{2}^{2}\right)} .
\end{gathered}
$$

The function $\varphi^{\prime} \mathrm{s}$ are given by

$$
\begin{aligned}
\varphi_{11}(y) & =-\frac{1}{1-y^{2}}+\frac{1}{\left(1-y^{2}\right)^{3 / 2}} \ln \left(\frac{1+\sqrt{1-y^{2}}}{y}\right) \theta(1-y)+ \\
& +\frac{1}{\left(y^{2}-1\right)^{3 / 2}} \arcsin \left(\frac{\sqrt{y^{2}-1}}{y}\right) \theta(y-1) ;
\end{aligned}
$$

$$
\begin{gathered}
\varphi_{12}(y)=\frac{1+y^{2}-2 y^{4}}{\left(1-y^{2}\right)^{3}}-\frac{3 y^{2}}{\left(1-y^{2}\right)^{5 / 2}} \ln \left(\frac{1+\sqrt{1-y^{2}}}{y}\right) \theta(1-y)- \\
-\frac{3 y^{2}}{\left(y^{2}-1\right)^{5 / 2}} \arcsin \left(\frac{\sqrt{y^{2}-1}}{y}\right) \theta(y-1) ;
\end{gathered}
$$

and

$$
\varphi_{22}(y)=\varphi_{11}(y)-\frac{\gamma_{12}}{3 \gamma_{2}} \varphi_{22}^{(1)}(y)+\frac{\gamma_{12}^{2}}{18 \gamma_{2}^{2}} \varphi_{22}^{(2)}(y)
$$

with

$$
\begin{gathered}
\varphi_{22}^{(1)}(y)=-\frac{4-5 y^{2}+y^{4}}{\left(1-y^{2}\right)^{3}}+ \\
+\frac{3}{\left(1-y^{2}\right)^{5 / 2}} \ln \left(\frac{1+\sqrt{1-y^{2}}}{y}\right) \theta(1-y)+ \\
+\frac{3}{\left(y^{2}-1\right)^{5 / 2}} \arcsin \left(\frac{\sqrt{y^{2}-1}}{y}\right) \theta(y-1), \\
+\frac{\varphi_{22}^{(2)}(y)=-\frac{19-25 y^{2}+8 y^{4}-2 y^{6}}{2\left(y^{2}+4\right)}}{2\left(1-y^{2}\right)^{7 / 2}} \ln \left(\frac{1+\sqrt{1-y^{2}}}{y}\right) \theta(1-y)- \\
-\frac{3\left(y^{2}+4\right)}{2\left(y^{2}-1\right)^{7 / 2}} \arcsin \left(\frac{\sqrt{y^{2}-1}}{y}\right) \theta(y-1) .
\end{gathered}
$$


1. Electrons on Helium and Other Cryogenic Substrates, E.Y. Andrei (ed.), Kluwer Academic Publ., Dordrecht (1997).

2. Yu.P. Monarkha and K. Kono, Two-Dimensional Coulomb Liquids and Solids, Springer, Berlin (2004).

3. P.M. Platzman and M.I. Dykman, Science 284, 1967 (1999).

4. T. Ando, J. Phys. Soc. Jpn. 44, 765 (1978).

5. D. Konstantinov, H. Isshiki, Yu.P. Monarkha, H. Akimoto, K. Shirahama, and K. Kono, Phys. Rev. Lett. 98, 235302-1 (2007).

6. Yu. Monarkha, D. Konstantinov, and K. Kono, Fiz. Nizk. Temp. 33, 942 (2007) [Low Temp. Phys. 33, 718 (2007)].

7. M.I. Dykman, P.M. Platzman, and P. Seddighrad, Phys. Rev. B67, 155402 (2003).

8. Yu.P. Monarkha, Fiz. Nizk. Temp. 4, 1093 (1978) [Sov. J. Low Temp. Phys. 4, 515 (1978)].

9. Yu.P. Monarkha and S.S. Sokolov, Fiz. Nizk. Temp. 32, 1278 (2006) [Low Temp. Phys. 32, 970 (2006)].

10. A. Lastri, F. Dalfovo, L.P. Pitaevskii, and S. Stringari, J. Low Temp. Phys. 98, 227 (1995).

11. H.J. Lauter, H. Godfrin, and P. Leiderer, J. Low Temp. Phys. 87, 425 (1992).

12. I.N. Adamenko, K.E. Nemchenko, and I.V. Tanatarov, J. Phys: Conf. Series 150, 032107 (2009).

13. D. Konstantinov and K. Kono, in: Proc. the 18th Intern. Conf. Electronic Properties of Two-Dimensional Systems (EP2DS-18); cond-mat. arXiv:0910.3040v2.
14. V.B. Shikin and Yu.P. Monarkha, J. Low Temp. Phys. 16, 193 (1974).

15. K.A. Nasyedkin, V.E. Sivokon, Yu.P. Monarkha, and S.S. Sokolov, Fiz. Nizk. Temp. 35, 968 (2009) [Low Temp. Phys. 35, 757 (2009)].

16. R.R. Gerhardts, Surf. Sci. 58, 227 (1976).

17. R. Kubo, H. Hasegawa, and N. Hashitsume, J. Phys. Soc. Jpn. 14, 56 (1959).

18. T. Ando and Y. Uemura, J. Phys. Soc. Jpn. 36, 959 (1974)

19. E. Rousseau, D. Ponarine, E. Varoquaux, and T. Mukharsky, J. Low Temp. Phys. 148, 193 (2007); E. Rousseau, D. Ponarine, L. Hristakos, O. Avenel, E. Varoquaux, and Y. Mukharsky, Phys. Rev. B79, 045406 (2009).

20. S.S. Sokolov, J.M. Villas-Bôas, Yu.P. Monarkha, and N. Studart, Fiz. Nizk. Temp. 34, 480 (2008) [Low Temp. Phys. 34, 385 (2008)].

21. Yu.Z. Kovdrya and Yu.P. Monarkha, Fiz. Nizk. Temp. 12, 1011 (1986) [Sov. J. Low Temp. Phys. 12, 571 (1986)]; A.V. Smorodin, V.A. Nikolaenko, and Yu.Z. Kovdrya, Pis'ma Zh. Eksp. Teor. Fiz. 73, 526 (2001) [JETP Letters 73, 465 (2001)].

22. S.S. Sokolov and O.I. Kirichek, Fiz. Nizk. Temp. 20, 764 (1994) [Low Temp. Phys. 20, 599 (1994)]. 